\title{
Effects of ampoule rotation on vertical zone-melting crystal growth: steady rotation versus accelerated crucible rotation technique (ACRT)
}

\author{
C.W. Lan*, J.H. Chian \\ Chemical Engineering Department, National Taiwan University, Taipei 10617, Taiwan, ROC \\ Received 23 November 1998; accepted 4 February 1999 \\ Communicated by D.T.J. Hurle
}

\begin{abstract}
Computer simulation is performed to illustrate the significance of ampoule rotation on the flows, interface shapes, and the growth rate in vertical zone-melting (VZM) crystal growth. For the growth of a $2 \mathrm{~cm}$ GaAs crystal in a quartz ampoule, simulation results show that even low-speed ampoule rotation can significantly affect the flows and further the growth interface. The concave growth front due to buoyancy convection can be inverted easily to a convex one by steady ampoule rotation. The accelerated crucible rotation technique (ACRT) also has a similar effect on the growth interface, but less effective. In addition, severe periodic growth and remelting is induced by ACRT, and it is enhanced significantly by natural convection. (C) 1999 Elsevier Science B.V. All rights reserved.
\end{abstract}

PACS: 44.25. + f; 47.27.Te; 81.10.Fq; 02.60.cb; 02.70.Fj

Keywords: Ampoule rotation; Zone-melting; Interface control; Buoyancy convection; Growth rate; ACRT

\section{Introduction}

The control of melt flow, the interface shape, and the growth rate is important in crystal growth processes. Especially, for crystal growth in an ampoule, a flat or convex growth front is usually required to minimize the parasitic nucleation of grains from the ampoule wall [1]. The convection and its associated heat and mass transfer are key factors for the

\footnotetext{
* Corresponding author. Fax: + 886-2-2363-3917.

E-mail address: lan@ruby.che.ntu.edu.tw (C.W. Lan)
}

interface control. Therefore, finding suitable operation parameters for a better control the transport processes is important for crystal growth. In addition to the interface shape, the melt flow is also responsible for the growth rate (solidification or melting) and dopant mixing. An unstable growth rate due to time-dependent flows and unfavorable solute segregation are also detrimental to crystal quality. Tuning heating profiles and applying magnetic fields are well-known approaches for controlling the melt flow [2-6]. Vibration can also be used $[7,8]$. For vertical Bridgman (VB) or zone-melting 
(VZM) crystal growth, the control of the interface shape or solute segregation by these approaches has been studied for years [e.g., Refs. [4-8]]. However, the effects of ampoule rotation, especially, steady rotation, have not been investigated until recently by Lan [9] for the VB configuration. He used ampoule rotation to damp the buoyancy flow and further to achieve the control of dopant segregation from a nearly complete mixing to diffusionlimited region. However, the interface shape and the growth rate were not affected much by ampoule rotation in his study.

In general, ampoule rotation is often used for (1) achieving an axisymmetric heating by steady rotation and (2) enhancing mixing by the accelerated crucible rotation technique (ACRT) [10]. The idea of ACRT is to rotate the ampoule or crucible at varying rates about the vertical axis. As the rotation rate changes, a momentum boundary layer, the so-called Ekman layer, is built up driving the melt flow either spin-up or spin-down. The effectiveness of ampoule rotation on crystal growth depends on several factors including the rotation speed, the system diameter, and the inherent flow structures and intensity, etc. For example, because the buoyancy flow in VB crystal growth is usually weak due to its stabilized thermal condition, a rotation speed of several tens of RPM may be enough to damp the flow [9]. In VZM crystal growth, due to radial heating, the buoyancy convection is much stronger and often causes a concave growth interface [11]. The damping of the buoyancy flow in VZM by the steady ampoule rotation may not be effective. On the contrary, the Ekman flow induced by ACRT may be dominant in the molten zone [12]. Therefore, with the combination of spin-up and spindown Ekman flow cycles, ACRT has been widely adopted for enhancing mixing and thus mass transfer. As a result, faster crystal growth from the solution without solvent inclusion may be possible $[13,14]$. Besides, ACRT can also have effects on the growth interface. A flatter growth front due to ACRT may be possible $[12,15]$, and this was also observed by Bloedner et al. [16] during the growth of $\mathrm{Hg}_{x} \mathrm{Cd}_{1-x} \mathrm{Te}$. In fact, the use of ACRT in crystal growth is still controversial. ACRT-induced growth striations have also been reported [17]. However, the theoretical study of ACRT for crystal growth is not adequate. Recently, some numerical investigations of ACRT have been reported $[12,15,18]$, as well as the extensive review of simplified models [19]. Unfortunately, these studies were based on the simplified models that neglected the growth front and the growth rate. Therefore, it has not been discussed how the growth rate and interface are affected by ACRT. Furthermore, the effects of steady ampoule rotation, though a simple operation, on the heat flow and the growth interface have not been investigated as well. As will be illustrated shortly, the steady ampoule rotation could be effective in the control of the flow and thus the growth interface, while keeping the growth stable.

In this paper, some significant aspects of ampoule rotation will be illustrated through numerical simulation. Using the growth of GaAs in a quartz ampoule as a model system, we will first illustrate how the steady ampoule rotation affects the flow locally and globally, which leads to an inversion of the growth interface. Furthermore, the effects of ACRT with the same rotation speed, e.g. 5 RPM, will then be discussed for the cases with and without the buoyancy force. In addition to the illustration of the flows, the growth front shape and the growth rate will be focused. In the next section, the mathematical model and its numerical solution are briefly described. To avoid misleading from possible numerical mistakes, we compare first our results with those from a commercial CFD package, Fluent, for a simplified model with steady rotation. Further comparison for a low-speed ACRT with analytical results will be performed before moving into the results and discussion in Section 3. Finally, brief conclusions are given in Section 4.

\section{Model and numerical solution}

The schematic of the VZM crystal growth used in this study is depicted in Fig. 1a. The furnace is described by an effective heating profile $T_{\mathrm{a}}(z, t)$, which is specified in modeling. To start crystal growth from a stationary state, this profile is moved upward at speed $U_{\mathrm{h}}$. The ampoule rotation speed $\Omega(t)$ can be constant or time-dependent. For the cases that a steady state can be achieved, the pseudo-steady-state model is adopted [20], which 


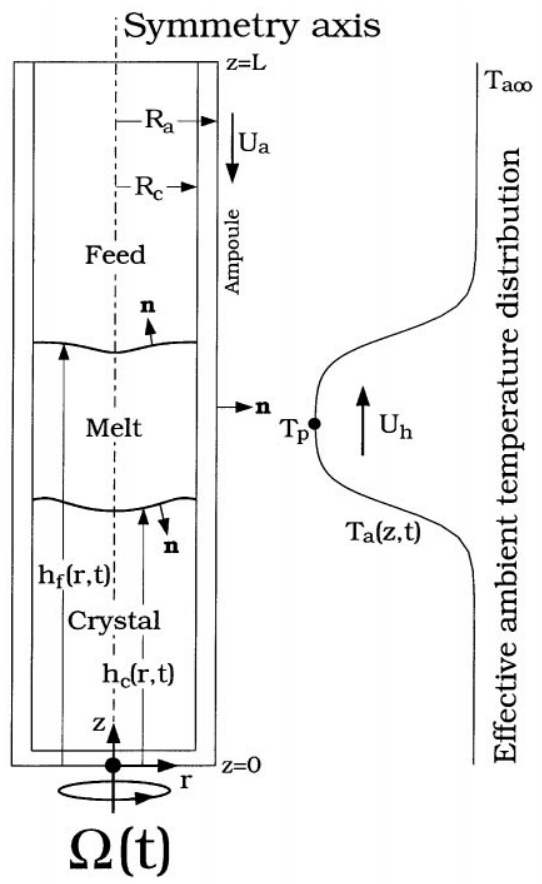

(a)

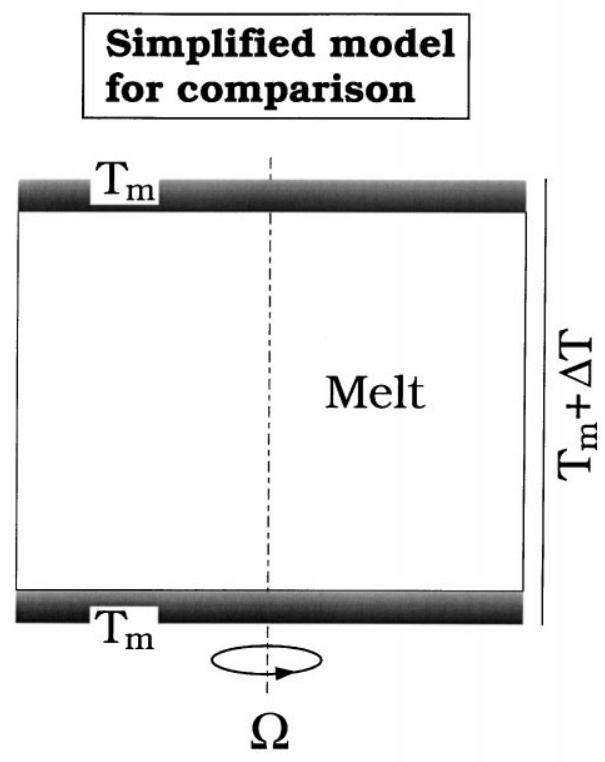

(b)

Fig. 1. Schematic sketch of vertical zone-melting (VZM) crystal growth: (a) full model; (b) simplified model.

saves computing time significantly. Therefore, for pseudo-steady-state calculations, the heating profile is kept stationary and the ampoule is moving downward at speed $U_{\mathrm{a}}$. Usually, near the middle of the ampoule, a pseudo-steady-state can be reached easily in $15 \mathrm{~min}$; we will illustrate that shortly. Therefore, for the cases that a pseudo-steady-state is possible, we will use the model for illustration. The system is assumed axisymmetric, so that the flow and temperature fields, as well as the growth front (the melt/crystal interface, $h_{\mathrm{c}}(r, t)$ ), and the feed $/$ melt interface, $h_{\mathrm{f}}(r, t)$, are represented in a cylindrical coordinate system $(r, z)$.

The melt is further assumed incompressible and Newtonian, while the flow is laminar. The Boussinesq approximation is also adopted. If the stream function $\psi$ and vorticity $\omega$ are defined in terms of radial $(u)$ and axial $(v)$ velocities as

$u=-\frac{1}{\rho_{\mathrm{m}} r} \frac{\partial \psi}{\partial z}, \quad v=\frac{1}{\rho_{\mathrm{m}} r} \frac{\partial \psi}{\partial r}$, and

$\omega=\frac{\partial u}{\partial z}-\frac{\partial v}{\partial r}$

the unsteady-state governing equations for stream function, vorticity, azimuthal velocity $\left(v_{\theta}\right)$, and temperature $(T)$ in the conservative-law form can be written as follows:

Equation of motion:

$$
\begin{gathered}
-\rho_{\mathrm{m}} \frac{\partial \omega}{\partial t}+\frac{\partial}{\partial r}\left(\frac{\omega}{r} \frac{\partial \psi}{\partial z}\right)-\frac{\partial}{\partial z}\left(\frac{\omega}{r} \frac{\partial \psi}{\partial r}\right) \\
+\frac{\partial}{\partial r}\left(\frac{1}{r} \frac{\partial}{\partial r}\left(\mu_{\mathrm{m}} r \omega\right)\right)+\frac{\partial}{\partial z}\left(\frac{1}{r} \frac{\partial}{\partial z}\left(\mu_{\mathrm{m}} r \omega\right)\right) \\
-\rho_{\mathrm{m}} \beta_{\mathrm{T}} g \frac{\partial T}{\partial r}+\rho_{\mathrm{m}} \frac{\partial}{\partial z}\left(\frac{v_{\theta}^{2}}{r}\right)=0 .
\end{gathered}
$$


Circulation equation:

$$
\begin{gathered}
-\rho_{\mathrm{m}} \frac{\partial v_{\theta}}{\partial t}+\frac{1}{r^{2}}\left[\frac{\partial}{\partial r}\left(r v_{\theta} \frac{\partial \psi}{\partial z}\right)-\frac{\partial}{\partial z}\left(r v_{\theta} \frac{\partial \psi}{\partial r}\right)\right] \\
+\frac{\partial}{\partial r}\left(\frac{\mu_{\mathrm{m}}}{r} \frac{\partial}{\partial r}\left(r v_{\theta}\right)\right)+\frac{\partial}{\partial z}\left(\frac{\mu_{\mathrm{m}}}{r} \frac{\partial}{\partial z}\left(r v_{\theta}\right)\right)=0 .
\end{gathered}
$$

Stream equation:

$$
\frac{\partial}{\partial z}\left(\frac{1}{\rho_{\mathrm{m}} r} \frac{\partial \psi}{\partial z}\right)+\frac{\partial}{\partial r}\left(\frac{1}{\rho_{\mathrm{m}} r} \frac{\partial \psi}{\partial r}\right)+\omega=0
$$

Energy equation:

$$
\begin{aligned}
& -\rho_{i} C_{p} r \frac{\partial T}{\partial t}-\frac{\partial}{\partial r}\left(\rho_{i} C_{p i} r u T\right)-\frac{\partial}{\partial z}\left(\rho_{i} C_{p i} r v T\right) \\
& +\frac{\partial}{\partial z}\left(r k_{i} \frac{\partial T}{\partial z}\right)+\frac{\partial}{\partial r}\left(r k_{i} \frac{\partial T}{\partial r}\right)=0, i=(\mathrm{m}, \mathrm{f}, \mathrm{c}, \mathrm{a}),
\end{aligned}
$$

where $\mu_{\mathrm{m}}$ is the melt viscosity. Also, $\rho_{i}$ is the density, $C_{p i}$ the specific heat, and $k_{i}$ the thermal conductivity of phase $i$; $i$ represents the melt (m), the feed (f), the crystal (c), or the ampoule (a). In addition, $g$ is the gravitational acceleration and $\beta_{\mathrm{T}}$ the thermal expansion coefficient. The associated thermal Rayleigh $\left(\mathrm{Ra}_{\mathrm{T}}\right)$ and Taylor $(\mathrm{Ta})$ numbers are defined as follows:

$\mathrm{Ra}_{\mathrm{T}} \equiv \beta_{\mathrm{T}} R_{\mathrm{c}}^{3} g T_{\mathrm{m}} /\left(v_{\mathrm{m}} \alpha_{\mathrm{m}}\right) ; \quad \mathrm{Ta} \equiv \Omega^{2} R_{\mathrm{c}}^{4} / v_{\mathrm{m}}^{2}$,

where $T_{\mathrm{m}}$ is the melting temperature of the pure material, $v_{\mathrm{m}}=\mu_{\mathrm{m}} / \rho_{\mathrm{m}}$, and $\alpha_{\mathrm{m}}=k_{\mathrm{m}} /\left(\rho_{\mathrm{m}} C p_{\mathrm{m}}\right)$. The characteristic length $R_{\mathrm{c}}$ is the crystal radius as shown in Fig. 1a. All the physical properties used in $\mathrm{Ra}_{\mathrm{T}}$ and $\mathrm{Ta}$ are obtained at $T_{\mathrm{m}}$. The reason for using $T_{\mathrm{m}}$ as the characteristic temperature is for convenience, since the temperature difference $\Delta T$ in the melt zone is unknown a priori.

To solve the previous equations, boundary conditions need to be specified. Most boundary conditions can be found elsewhere [20,21]. In brief, the no-slip boundary condition for velocities is used at solid boundaries. For example, at the melt/solid interfaces,

$v_{\theta}=r \Omega(t)$,

where $\Omega(t)$ is the ampoule rotation speed.
Heat transfer from the system to the ambient is governed by convection and radiation:

$$
\begin{aligned}
-\left(\boldsymbol{n} k_{\mathrm{a}} \cdot \nabla T\right)= & h\left[T-T_{\mathrm{a}}(z, t)\right] \\
& +\varepsilon_{\mathrm{a}} \sigma\left[T^{4}-T_{\mathrm{a}}(z, t)^{4}\right],
\end{aligned}
$$

where $h$ is the heat transfer coefficient, $\varepsilon_{\mathrm{a}}$ the ampoule emissivity, and $\sigma$ the Stefan-Boltzmann constant. $T_{\mathrm{a}}(z, t)$ is the effective ambient temperature, which is described by a Fermi-like distribution:

$$
T_{\mathrm{a}}(z)=\frac{T_{\mathrm{p}}-T_{\mathrm{a} \infty}}{\exp \left\{\left(\left|z-z_{\mathrm{p}}(t)\right|-w\right) / a\right\}+1}+T_{\mathrm{a} \infty},
$$

and

$z_{\mathrm{p}}(t)=z_{\mathrm{p} 0}+\int_{0}^{t} U_{h} \mathrm{~d} t$,

where $T_{\mathrm{p}}$ and $T_{\mathrm{a} \infty}$ are the peak and background temperatures, respectively, the parameters $\omega$ and $a$ are related to the width of the distribution, $z_{\mathrm{p}}(t)$ is the position of $T_{\mathrm{p}}$, and $z_{\mathrm{p} 0}$ the initial position of $T_{\mathrm{p}}$. An example of this distribution is shown on the right of Fig. 1a, which is similar to that measured from a typical zone heater furnace [13]. The top surface of the system is assumed adiabatic, and there is not ampoule cover there. A more realistic boundary condition can be used if necessary.

The above governing equations and their associated boundary conditions can only be solved numerically. We have developed efficient finitevolume schemes using Newton's method with a solution tracking capability and the DASPK solver for integration [22] for solving these equations. A detailed description of the numerical method can be found elsewhere [20,21].

\section{Results and discussion}

Before showing calculated results, careful examination of the numerical methods is necessary for both steady- and unsteady-state calculations. Therefore, we have adopted a simplified model as shown in Fig. 1b for comparison purposes. The heating profile is constant and kept at $1711 \mathrm{~K}$. The physical properties of the materials are listed in Table 1 . The results calculated by a commercial code, Fluent, for different rotation speeds are shown 
Table 1

Physical properties and some input parameters [26]

\section{GaAs}

$\rho_{\mathrm{c}}=\rho_{\mathrm{f}}=5.7 \mathrm{~g} \mathrm{~cm}^{-3}$

$\rho_{\mathrm{m}}=5.7 \mathrm{~g} \mathrm{~cm}^{-3}$

$T_{\mathrm{m}}=1511 \mathrm{~K}$

$\Delta H=726 \mathrm{~J} \mathrm{~g}^{-1}$

$h=4.096 \times 10^{-4} \mathrm{~W} \mathrm{~cm}^{-2}{ }^{\circ} \mathrm{C}^{-1}$

$k_{\mathrm{c}}=k_{\mathrm{f}}=0.07 \mathrm{~W} \mathrm{~cm}^{-1}{ }^{\circ} \mathrm{C}^{-1}$

$k_{\mathrm{m}}=0.14 \mathrm{~W} \mathrm{~cm}^{-1}{ }^{\circ} \mathrm{C}^{-1}$

$C p_{\mathrm{c}}=C p_{\mathrm{f}}=C p_{\mathrm{m}}=0.42 \mathrm{~J} \mathrm{~g}^{-1}{ }^{\circ} \mathrm{C}^{-1}$

$\beta_{\mathrm{T}}=1.16 \times 10^{-4} \mathrm{~K}^{-1}$

\section{Quartz}

$\rho_{\mathrm{a}}=2.2 \mathrm{~g} \mathrm{~cm}^{-3}$

$k_{\mathrm{a}}=0.035 \mathrm{~W} \mathrm{~cm}^{-1}{ }^{\circ} \mathrm{C}^{-1}$

$C p_{\mathrm{a}}=0.188 \mathrm{~J} \mathrm{~g}^{-1}{ }^{\circ} \mathrm{C}^{-1}$

$\varepsilon_{\mathrm{a}}=0.7$

\section{Other input parameters}

$L=15 \mathrm{~cm}$

$R_{\mathrm{c}}=1 \mathrm{~cm}$

$R_{\mathrm{a}}=1.2 \mathrm{~cm}$

$T_{\mathrm{p}}=1800 \mathrm{~K}$

$T_{\mathrm{a} \infty}=898 \mathrm{~K}$

$w=1.25 \mathrm{~cm}$

$a_{\mathrm{f}}=a_{\mathrm{c}}=0.2 \mathrm{~cm}$

$z_{\mathrm{p} 0}=7.5 \mathrm{~cm}$

$\Omega_{0}=0-20 \mathrm{RPM}$

$\omega_{0}=0.02 \pi \mathrm{rad} / \mathrm{s}$

$U_{\mathrm{a}}=-1 \mathrm{~cm} / \mathrm{h}$ (for pseudo-steady-state claculations)

$U_{\mathrm{h}}=1 \mathrm{~cm} / \mathrm{h}$ (for fully transient calculations) in Fig. 2. The flow patterns are on the left and the isotherms are on the right; it is the same for the rest of field plots. The conditions used are also shown, and the detailed comparison with our numerical calculations is listed in the table. In fact, in Fluent, the calculation can be performed using either a fixed frame with rotating boundaries or a rotating frame. However, the calculated results are almost the same. With a similar mesh, $41 \times 61$ in the melt, our results are in good agreement with Fig. 2, as shown in the table. We have further performed mesh refinement for checking numerical errors, but the calculated results are not changed much. Therefore, the same mesh $(41 \times 61$ in the melt $)$ is used for all the calculations here. From Fig. 2a, it is obvious that the buoyancy convection is strong, so that the isotherms are highly distorted; the overheating in the melt is $53.69^{\circ} \mathrm{C}$. The highest temperature at the surface moves upward significantly due to the flow, and the downward flow near the symmetry axis pushes the isotherms downward. As will be shown later, such a flow often leads to a concave growth front, which is not favorable for growing single crystals. As the rotation speed is increased to 3 RPM in Fig. 2b, a small secondary cell is induced near the center of the growth front. As a result, the isotherms are now pushed upward slightly. Interestingly, the intensity of the main flow $\left(\left|\psi_{\text {min }}\right|\right)$ is not

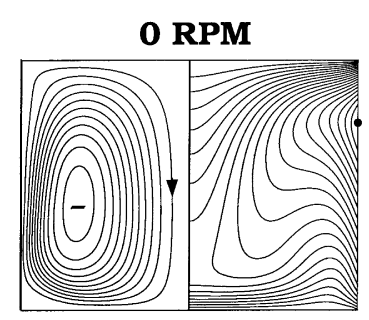

(a)

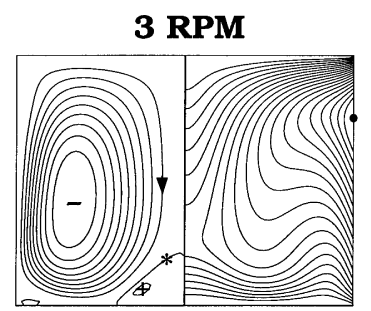

(b)

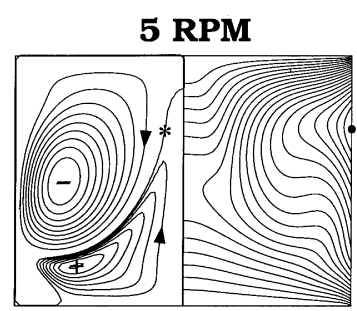

(c)

\begin{tabular}{|c|c|c|c|c|c|}
\hline & & O RPM & 3 RPM & 5 RPM & \multirow{5}{*}{$\begin{array}{l}\mathrm{T}_{\mathrm{a}}=1711 \mathrm{~K}, \\
\mathrm{~h}=160 \mathrm{~W} / \mathrm{m}^{2} \mathrm{~K}, \\
\varepsilon=0.7\end{array}$} \\
\hline \multirow{2}{*}{ Fluent } & $\mathrm{T}_{\max }(\mathrm{K})$ & 1564.69 & 1565.04 & 1568.84 & \\
\hline & $\Psi_{\min }(\mathrm{g} / \mathrm{s})$ & -1.083 & -1.128 & -1.250 & \\
\hline \multirow{2}{*}{ Present } & $\mathrm{T}_{\max }(\mathrm{K})$ & 1564.56 & 1565.56 & 1569.82 & \\
\hline & $\Psi_{\min }(\mathrm{g} / \mathrm{s})$ & -1.077 & -1.115 & -1.234 & \\
\hline
\end{tabular}

Fig. 2. Calculated flow patterns and isotherms using Fluent for the simplified model: (a) 0 RPM (Ta = 0); (b) 3 RPM (Ta=7.956); (c) $5 \mathrm{RPM}(\mathrm{Ta}=13.26)$. $\mathrm{Ra}_{\mathrm{T}}=8.51 \times 10^{5}$. The minimum stream function $\psi_{\min }$ is indicated by a minus $(-)$ ), $\psi=0$ by an asterisk $(*)$, $\psi_{\max }$ by an plus $(+)$, and $T_{\max }$ by $(\mathbf{)}$. Detailed comparison with our numerical results is illustrated in the table. 
damped by the rotation; also see the table in Fig. 2. On the contrary, its intensity increases due to the slightly higher overheating. The secondary flow cell continues to grow with the increasing rotation speed. As shown in Fig. 2c at 5 RPM rotation, the secondary cell becomes much larger, and again the isotherms near the growth front are pushed upward further more. The flow intensity is still not reduced at all by rotation. In fact, the main flow will be suppressed at much higher rotation speeds at which the overheating effect can be compensated. Nevertheless, from the distortion of the isotherms from (a) to (c), the heat transfer becomes more conductive with the increasing rotation speed, especially, near the growth front. In other words, from the isotherm patterns, it is clear that the interface control can be achieved by rotation. Furthermore, unlike the VB case shown by Lan [9], the global flow here is not suppressed effectively by ampoule rotation. Instead, the rotation modifies the local flow near the growth front, and thus affects the heat transfer and the interface there.

The second comparison is performed for ACRT using a long cylinder; its top and bottom boundaries are neglected. The system is also assumed isothermal. If the rotation speed is low, an analytical solution for a sinusoidal ampoule rotation, i.e., $\Omega(t)=\Omega_{0} \sin \left(\omega_{0} t\right)$, can be obtained (after the transient period) as the following [19]:

$$
\begin{aligned}
\frac{\Omega(r, t) r}{\Omega_{0} R_{c}}= & -\cos \left(\omega_{0} t\right) \\
& \times \frac{\operatorname{ber}^{\prime}(k r) \operatorname{bei}^{\prime}\left(k R_{\mathrm{c}}\right)-\operatorname{bei}^{\prime}(k r) \operatorname{ber}^{\prime}\left(k R_{\mathrm{c}}\right)}{\left(\operatorname{ber}^{\prime}\left(k R_{\mathrm{c}}\right)\right)^{2}+\left(\operatorname{bei}^{\prime}\left(k R_{\mathrm{c}}\right)\right)^{2}} \\
& +\sin \left(\omega_{0} t\right) \\
& \times \frac{\operatorname{ber}^{\prime}(k r) \operatorname{ber}^{\prime}\left(k R_{\mathrm{c}}\right)+\operatorname{bei}^{\prime}(k r) \operatorname{bei}^{\prime}\left(k R_{\mathrm{c}}\right)}{\left(\operatorname{ber}^{\prime}\left(k R_{\mathrm{c}}\right)\right)^{2}+\left(\operatorname{bei}^{\prime}\left(k R_{\mathrm{c}}\right)\right)^{2}},
\end{aligned}
$$

where $k^{2}=\omega_{0} / v_{\mathrm{m}}$ and $\operatorname{ber}^{\prime}(x)$ and bei' $^{\prime}(x)$ are Kelvin functions [23], which are evaluated by the mathematical library NAG [24]. As the initial transient period has passed (it takes about two to three cycles), the rotation speed in the melt changes periodically with ampoule rotation. The comparison of our calculations with the analytical results at differ-

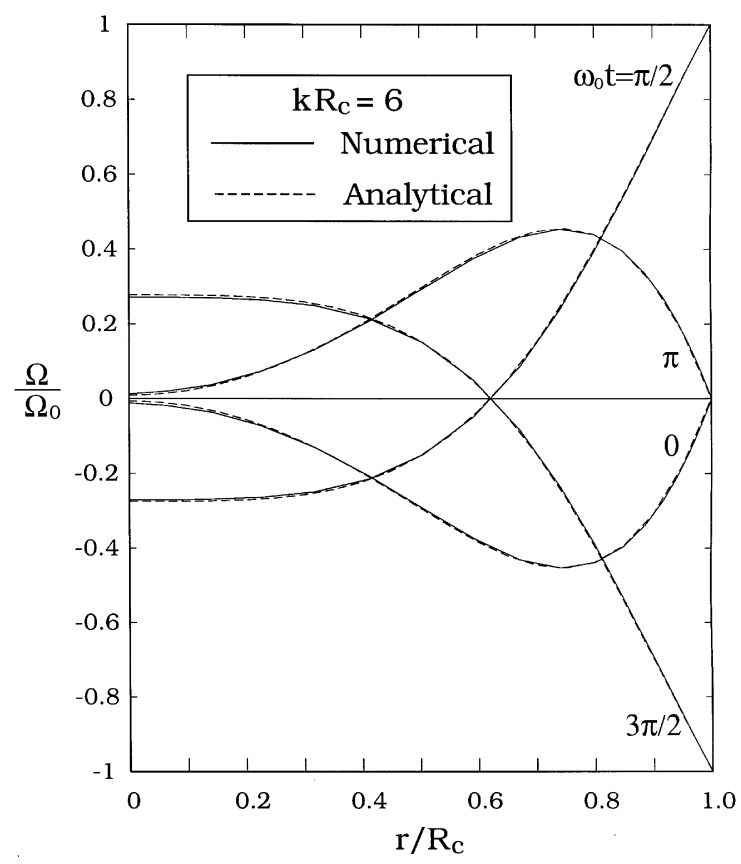

Fig. 3. Comparison of the calculated angular velocity distribution (solid lines) with analytical solutions (dashed lines) for an infinitely long cylinder with a sinusoidal rotation.

ent times of a steady cycle is shown in Fig. 3. As shown, excellent agreement is obtained as well. Other comparisons for spin-up (by increasing the rotation speed from zero to a finite speed) and inertial-internal gravity waves [25], which cause the oscillation of the angular speed in the fluid, for a finite cylinder are also conduced. Good agreement with the analytical or experimental results are obtained.

After building the confidence on the numerical methods, we are now ready to illustrate our calculated results for the VZM growth of a $2 \mathrm{~cm}$ GaAs crystal in a quartz ampoule. Detailed physical and input data are listed in Table 1 [26].

\subsection{Effects of steady ampoule rotation}

The effects of 5 RPM steady ampoule rotation for different convection levels are shown in Fig. 4. Without ampoule rotation, as shown from Fig. 4a to Fig. 4c, it is clear that the natural convection enhances the heat transfer and causes a concave 


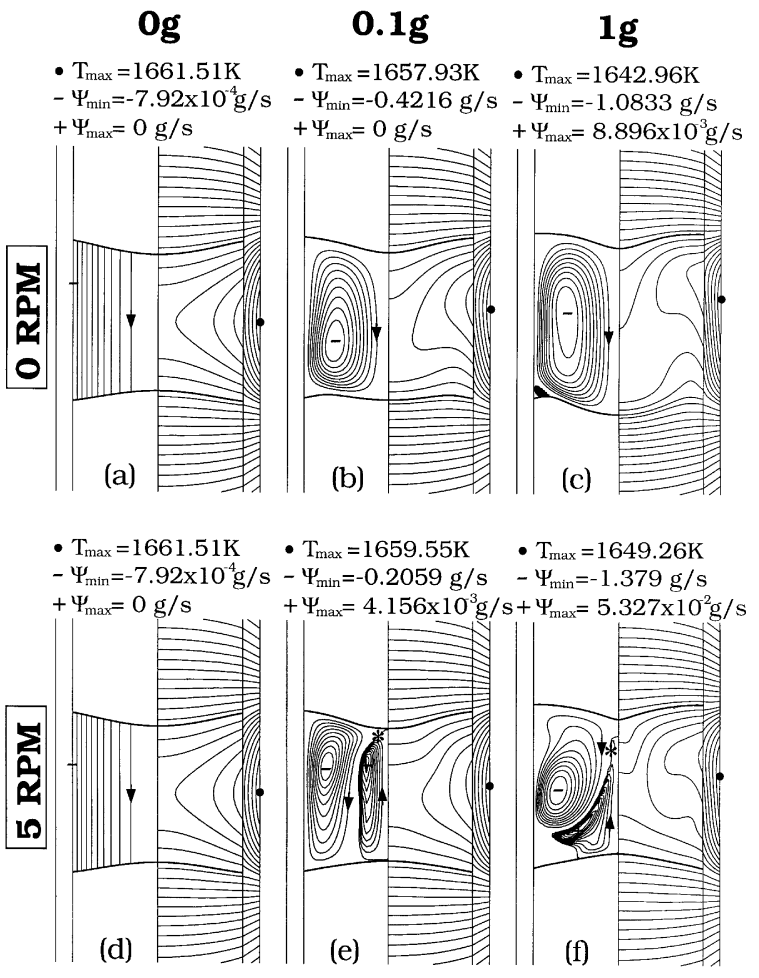

Fig. 4. Effects of buoyancy convection and 5 RPM ampoule rotation on the buoyancy flow and interface shapes.

growth front; the zone length also increases and the melt overheating decreases with the increasing $g$. With 5 RPM ampoule rotation, as shown from Fig. $4 \mathrm{~d}$ to Fig. 4f, there is no effect for $0 \mathrm{~g}\left(\mathrm{Ra}_{\mathrm{T}}=0\right)$. However, for $0.1 \mathrm{~g}\left(\mathrm{Ra}_{\mathrm{T}}=8.51 \times 10^{4}, \mathrm{Ta}=13.26\right)$ in Fig. 4e, the intensity of the buoyancy flow is reduced (from 0.4216 to $0.2059 \mathrm{~g} / \mathrm{s}$ ). Meanwhile, a secondary flow cell near the growth front is induced. The distortion of the isotherms is also reduced significantly. As a result, the growth interface becomes convex. At normal gravity $\left(\mathrm{Ra}_{\mathrm{T}}=8.51 \times 10^{5}, \mathrm{Ta}=13.26\right)$ in Fig. $4 \mathrm{f}$, the main flow intensity is not reduced but enhanced, and this is mainly due to the higher thermal gradients caused by the increase of overheating as compared to 0 RPM. However, near the growth front, a much larger secondary cell is induced. Due to the secondary cell, the isotherms are now pushed upward slightly. As a result, a convex growth front is obtained. At the beginning of this research, we were

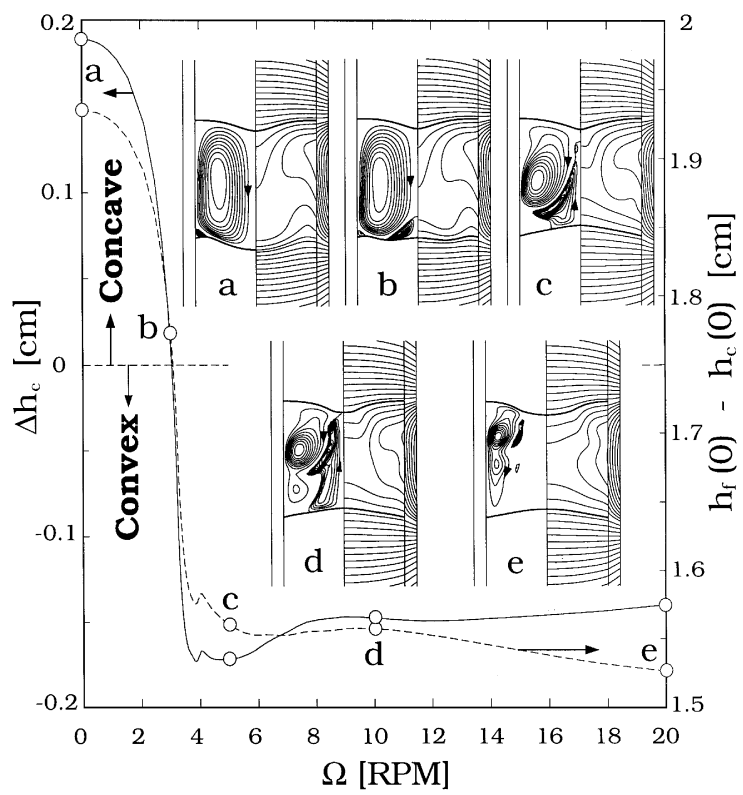

Fig. 5. Effect of ampoule rotation on the interface deflection $\left(\Delta h_{\mathrm{c}}\right)$, zone length $\left(h_{\mathrm{f}}-h_{\mathrm{c}}\right)$, and heat flows. The heat flow patterns from a to e are corresponding to $0,3,5,10$, and 20 RPM ampoule rotation, respectively. $\psi_{\min }=-1.0833, \psi_{\max }=8.896 \times$ $10^{-3} \mathrm{~g} / \mathrm{s}$, and $T_{\max }=1642.96 \mathrm{~K}$ for $a ; \psi_{\min }=-1.1012$, $\psi_{\max }=2.650 \times 10^{-2} \mathrm{~g} / \mathrm{s}$, and $T_{\max }=1643.85 \mathrm{~K}$ for $b ; \psi_{\min }=$ $-1.2788, \psi_{\max }=5.327 \times 10^{-2} \mathrm{~g} / \mathrm{s}$, and $T_{\max }=1649.26 \mathrm{~K}$ for $c$; $\psi_{\min }=-1.286, \psi_{\max }=2.378 \times 10^{-2} \mathrm{~g} / \mathrm{s}$, and $T_{\max }=1650.21 \mathrm{~K}$ for $d ; \psi_{\min }=-0.8059, \psi_{\max }=1.0493 \times 10^{-2} \mathrm{~g} / \mathrm{s}$, and $T_{\max }=$ $1653.87 \mathrm{~K}$ for e.

cautious about the correctness of the results because $5 \mathrm{RPM}$ rotation is usually believed to be too small to have any significance on the crystal interface. However, after extensive benchmark testing and comparison with other numerical methods, as well as the commercial code, Fluent, we are quite confident with the results. Such results provide new information for crystal growers to reexamine the significance of ampoule rotation. According to the calculations, the ampoule rotation, even at a low speed, could be significant on the flows, heat transfer, and the growth front shape. Again, the modification of the local heat flow near the growth front is the key to make it work.

Further illustration is also conducted for normal gravity. We trace our solution from 0 to 20 RPM, and the results are summarized in Fig. 5. The 
deflection of the growth front $\Delta h_{\mathrm{c}} \equiv h_{\mathrm{c}}\left(R_{\mathrm{c}}\right)-h_{\mathrm{c}}(0)$ is shown on the left vertical axis, while the zone length at the centerline $h_{\mathrm{f}}(0)-h_{\mathrm{c}}(0)$ on the right. As shown, the dramatic change of the growth front shape, from concave to convex, occurs at about 3 RPM, where the secondary cell appears; also see the flow patterns and isotherms in Fig. 5b. After 5 RPM rotation, the change of the interface deflection and zone length is not much. However, at higher rotation speed, say 20 RPM, the main flow intensity starts to decrease. At high rotation speed, the damping effect is also effective near the melt core because the heat transfer there is changed from convective to conductive modes. The TaylorProudman column [27] near the melt core becomes obvious after 10 RPM. Such a change can also be seen from the distortion of the isotherms. However, as far as the interface control is concerned, only the heat transfer near the growth front needs to be modified, and slow rotation seems to be enough to achieve that. Furthermore, for radial solute segregation, the appearance of the secondary cell at 3 RPM is believed to be important as well. Indeed, both cases require only the modification of the local flow near the growth front, and that is why the slow rotation works in this study.

Furthermore, the time to achieve a pseudosteady growth for both 0 and 5 RPM is about the same. We have also conducted transient calculations for both cases, as shown in Fig. 6. The initial

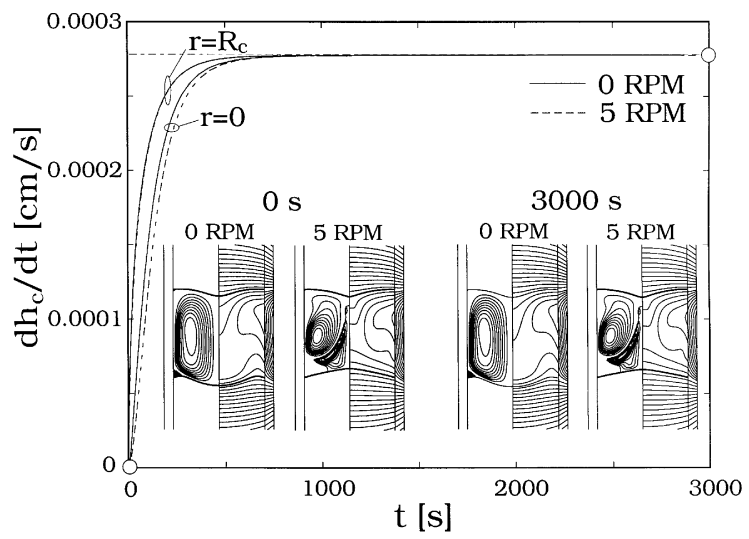

Fig. 6. Dynamic response of the growth rates $\left(r=0\right.$ and $\left.r=R_{\mathrm{c}}\right)$ from stationary to a pseudo-steady-state growth at $U_{\mathrm{h}}=1 \mathrm{~cm} / \mathrm{h}$ for 0 and 5 RPM ampoule rotation. stationary conditions are obtained at $U_{\mathrm{a}}=U_{\mathrm{h}}=0$. At $t=0$, we change $U_{\mathrm{h}}$ to $1 \mathrm{~cm} / \mathrm{h}$. As shown, the growth rates at both $r=0$ and $R_{\mathrm{c}}$ take less than 15 min to reach a steady growth. Furthermore, because the growth rate is not high, by comparing the zone shapes at $t=0$ and $3000 \mathrm{~s}$, its effect on the growth front is small. More importantly, the slow ampoule rotation does not seem to introduce any flow instability to the growth in this case.

\subsection{Unsteady ampoule rotation - ACRT}

Another way, which is a well-known technique to control the flows and heat transfer is ACRT. Therefore, a growth simulation for ACRT is performed. We also use 5 RPM for illustration, and the sinusoidal ampoule rotation is used. Other rotation cycles may be used, but we believe that their effects will be similar.

We start from a steady growth result (without rotation) from the previous figure (Fig. 6) at $t=3000 \mathrm{~s}$, which is now reset to $t=0$. At $t=0$, we turn on the ampoule rotation, as shown on the top of Fig. 7a; the period is $100 \mathrm{~s}$ and the amplitude is 5 RPM. Again, as shown from Fig. 7a-Fig. 7c, the system takes about 8 min to reach a pseudo-steady periodic state. However, as shown in Fig. 7a, the amplitude of the oscillatory interface speeds (growth and remelting) is much larger than the preset growth rate, $1 \mathrm{~cm} / \mathrm{h}$ (or $2.7778 \times 10^{-4} \mathrm{~cm} / \mathrm{s}$ ), which is also indicated by a dashed line above zero. In practice, such severe growth and remelting can be a source of growth striations.

The zone length also changes with the rotation speed, as shown in Fig. $7 \mathrm{~b}$; the mean length is reduced by ACRT. More importantly, the interface deflection $\Delta h_{\mathrm{c}}$ in Fig. 7c is also reduced by ACRT, which is similar to that due to 5 RPM steady rotation, but less effective. If we take one period from $t=500$ to $600 \mathrm{~s}$, the system response can be examined closely, as illustrated in Fig. 8. At $t=500 \mathrm{~s}$, the flow is at the end of a spin-down cycle, and the Ekman flow near the growth front has the same flow direction as the natural convection. Therefore, its effect is not obvious. However, at the end of spin-up at $t=525 \mathrm{~s}$, the Ekman flow cell near the growth front, which brings the heat away from the growth front, causes the faster 


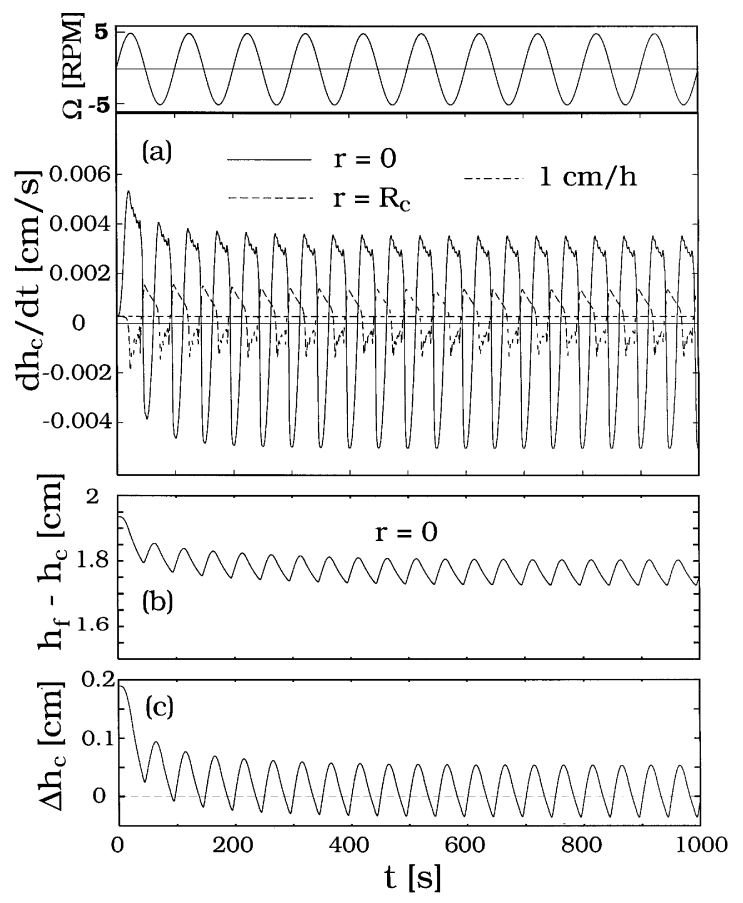

Fig. 7. Effect of 5 RPM ACRT on (a) growth rates $(r=0$ and $r=R_{\mathrm{c}}$ ); (b) zone length at $r=0$; (c) interface deflection. The rotation cycle is shown on the top of the figure. $\mathrm{Ra}_{\mathrm{T}}$ $=8.51 \times 10^{5}$.

growth near the centerline as well as the upward distortion of the isotherms there. Meanwhile, the interface near the ampoule surface is melted back. At the end of spin-down $(t=550 \mathrm{~s})$, the interface at the centerline is melted back, while the crystal grows near the ampoule wall. Because one period consists of two cycles of spin-up and spin-down, the second cycle (from 550 to $600 \mathrm{~s}$ ) is almost the same as the first cycle. Furthermore, from the flow patterns, it is clear that ACRT only affects the flow locally near the growth interface. Therefore, its effect on the feed front is much less. In addition, its effects on the shape of the growth interface is not as effective as that by steady ampoule rotation. Nevertheless, the severe growth and remelting being much larger than the growth rate is not expected, but it may be interesting to crystal growers. As will be illustrated shortly, for 5 RPM ACRT without the buoyancy force, the Ekman flow alone cannot generate such a severe effect, and this unveils the role of natural convection in ACRT.

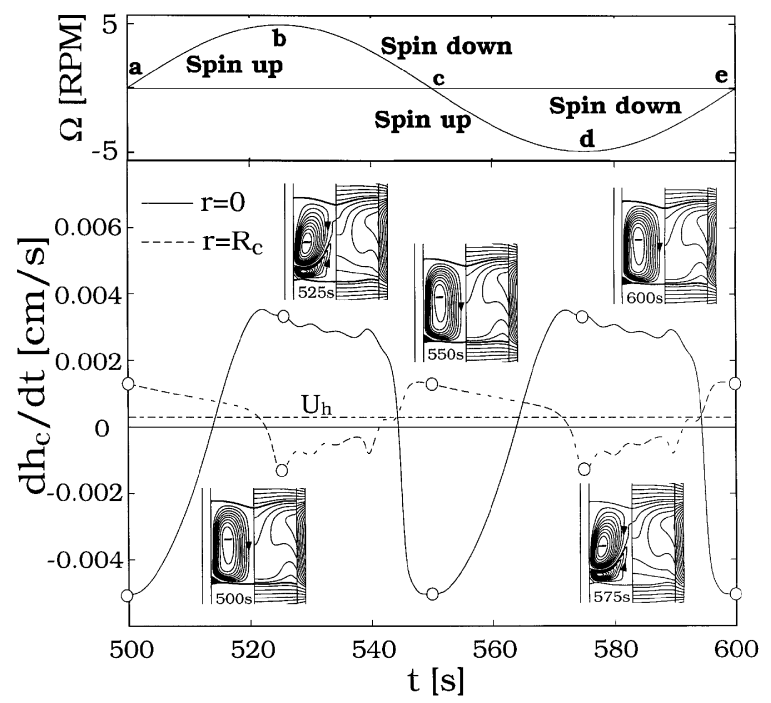

Fig. 8. One period of ampoule rotation cycle from $t=500$ to $600 \mathrm{~s}: \psi_{\min }=-1.0865, \psi_{\max }=1.425 \times 10^{-2} \mathrm{~g} / \mathrm{s}$, and $T_{\max }=$ $1643.76 \mathrm{~K}$ for $t=500 \mathrm{~s} ; \psi_{\min }=-1.294, \psi_{\max }=0.1750 \mathrm{~g} / \mathrm{s}$, and $T_{\max }=1646.79$ for $t=525 \mathrm{~s} ; \psi_{\min }=-1.0866, \psi_{\max }=1.451 \times$ $10^{-2} \mathrm{~g} / \mathrm{s}$, and $T_{\max }=1643.74 \mathrm{~K}$ for $t=550 \mathrm{~s} ; \psi_{\min }=-1.2927$, $\psi_{\max }=0.1729 \mathrm{~g} / \mathrm{s}$, and $T_{\max }=1646.79 \mathrm{~K}$ for $t=575 \mathrm{~s}$; $\psi_{\min }=-1.0866, \psi_{\max }=1.451 \times 10^{-2} \mathrm{~g} / \mathrm{s}$, and $T_{\max }=1643.74 \mathrm{~K}$ for $t=600 \mathrm{~s}$.

For the case with the same ACRT cycle, but without buoyancy force, the calculated results are shown in Fig. 9. As shown in Fig. 9a, the oscillation amplitude of the growth rate, as compared with Fig. 7a, decreases significantly. However, the maximum growth speed is still about two times of the present speed and the remelting still occurs occasionally. The change in the zone length (Fig. 9b) and the interface deflection (Fig. 9c) is reduced slightly. The system response from 500 to $600 \mathrm{~s}$ is shown in Fig. 10. As shown, typical Ekman flows due to spin-up and spin-down are revealed; again (a) is at the end of one spin-down cycle. However, because the rotation speed is only 5 RPM, their effects on the heat transfer and thus the interface shape are not as obvious as in the previous cases. Clearly, buoyancy convection plays an important role during ACRT at slow rotation. Although the Ekman flow is weak at 5 RPM, its periodic disturbance to the main buoyancy cell could cause severe growth and remelting. Such a mechanism is very 

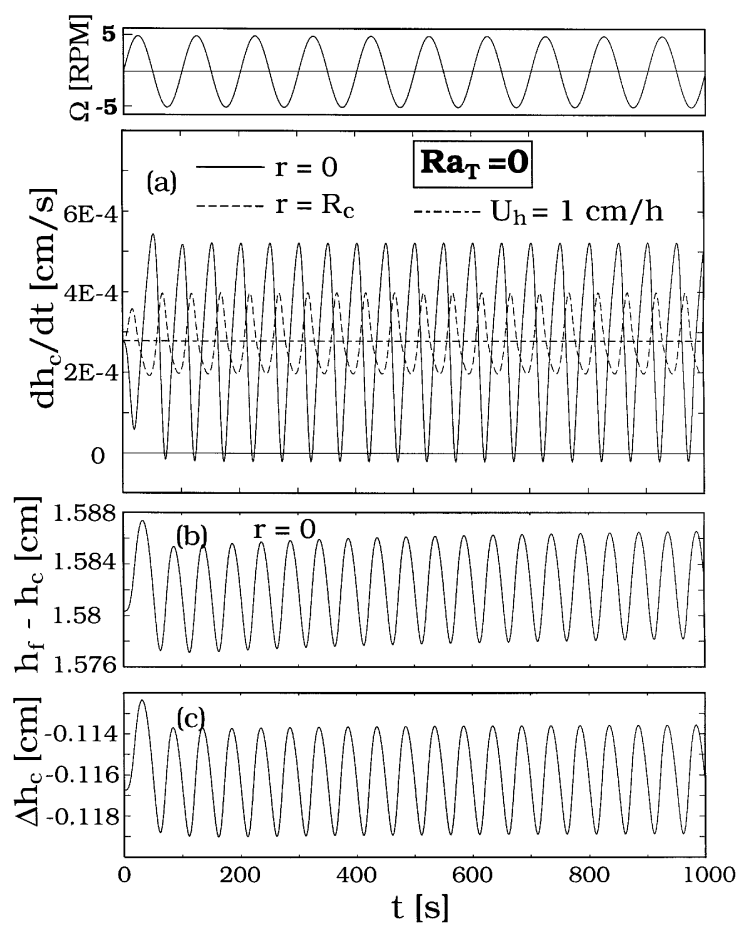

Fig. 9. Effect of 5 RPM ACRT without buoyancy force $\left(\mathrm{Ra}_{\mathrm{T}}=0\right)$ : (a) growth rates; (b) zone length; (c) interface deflection.

different from that of a typical ACRT at several tens of RPM, in which the Ekman flow dominates over the buoyancy flow [12].

From the crystal growth point of view, the severe growth and remelting induced by ACRT usually is not favorable. However, ACRT could be an effective way to generate regular striation patterns that may be useful, for example, in the generation of quasi-phase matching domain for nonlinear optical crystals.

\section{Conclusions}

The effects of ampoule rotation, both steady rotation and ACRT, on the flows, heat transfer, the growth rate, and the growth front shape are studied by numerical simulation for vertical zone-melting crystal growth. It is found for the first time that the steady ampoule rotation, even at a low speed of 3-5 RPM, can significantly affect the heat flow and the interface shape. Interface inversion from concave to

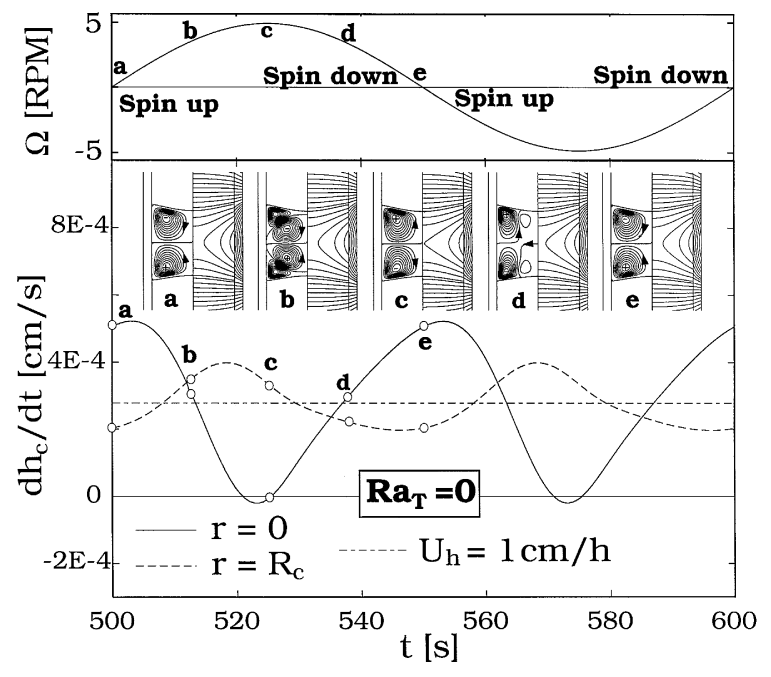

Fig. 10. One period of ampoule rotation cycle from $t=500$ to $600 \mathrm{~s}$ without buoyancy force: $\psi_{\min }=-1.924 \times 10^{-2}$, $\psi_{\max }=1.928 \times 10^{-2} \mathrm{~g} / \mathrm{s}$, and $T_{\max }=1661.42 \mathrm{~K}$ for a $(t=500 \mathrm{~s})$; $\psi_{\min }=-9.344 \times 10^{-3}, \quad \psi_{\max }=9.536 \times 10^{-3} \mathrm{~g} / \mathrm{s}$, and $T_{\max }=$ $1661.39 \mathrm{~K} \quad$ for $\mathrm{b} \quad(t=512.5 \mathrm{~s}) ; \quad \psi_{\min }=-1.878 \times 10^{-2}$, $\psi_{\max }=1.894 \times 10^{-2} \mathrm{~g} / \mathrm{s}$, and $T_{\max }=1661.68 \mathrm{~K}$ for $\mathrm{c}(t=525 \mathrm{~s})$; $\psi_{\min }=-2.787 \times 10^{-3}, \quad \psi_{\max }=2.865 \times 10^{-3} \mathrm{~g} / \mathrm{s}$, and $T_{\max }=$ $1661.62 \mathrm{~K}$ for $\mathrm{d} \quad(t=537.5 \mathrm{~s}) ; \quad \psi_{\min }=-1.924 \times 10^{-2}$, $\psi_{\max }=1.928 \times 10^{-2} \mathrm{~g} / \mathrm{s}$, and $T_{\max }=1661.42 \mathrm{~K}$ for e $(t=550 \mathrm{~s})$.

convex is achieved. The local flow near the growth front induced by the centrifugal force plays a key role in the heat flow and thus the interface control. With the same rotation speed, ACRT has similar effects, but less effective. ACRT also induces severe growth and remelting that may be responsible for growth striations. Interestingly, the amplitude of the oscillatory speed is reduced significantly if the buoyancy force is removed. Although the numerical calculations are carefully performed, the confirmation of the effects of ampoule rotation still requires crystal growth experiments. Nevertheless, the present calculations may provide useful information, from the theoretical side, to crystal growers in the control of the interface shape and the growth rate by ampoule rotation.

\section{References}

[1] J.C. Brice, Crystal Growth Processes, Wiley, New York, 1986. 
[2] C.W. Lan, C.C. Ting, Int. J. Numer. Meth. Heat Tranfer Fluid Flow 6 (1996) 3.

[3] D. Hofmann, T. Jung, G. Muller, J. Crystal Growth 128 (1993) 213.

[4] H.A. Chedzey, D.T.J. Hurle, Nature 210 (1966) 933.

[5] H.P. Utech, M.C. Flemming, J. Appl. Phys. 37 (1966) 2021.

[6] D.H. Kim, P.M. Adornato, R.A. Brown, J. Crystal Growth 89 (1988) 339.

[7] X.F. Shen, A.V. Anilkumar, R.N. Grugel, T.G. Wang, J. Crystal Growth 165 (1996) 165.

[8] D.V. Lyubimov, T.P. Lyubimova, S. Meradji, B. Roux, J. Crystal Growth 180 (1997) 648.

[9] C.W. Lan, Effects of Ampoule Rotation on the Flows and Dopant Segregation in Vertical Bridgman Crystal Growth, J. Crystal Growth 197 (1999) 983.

[10] H.J. Scheel, E.O. Shulz-Dubois, J. Crystal Growth 8 (1971) 304.

[11] C.W. Lan, D.T. Yang, Modeling Simul. Mater. Sci. Eng. 3 (1995) 71.

[12] R.U. Barz, P. Sabhapathy, M. Salcudean, J. Crystal Growth 180 (1997) 566.

[13] F.V. Wald, R.O. Bell, J. Crystal Growth 30 (1975) 29.

[14] R.U. Bloedner, P. Gille, J. Crystal Growth 10 (1993) 181.
[15] S.J. Moon, C.J. Kim, S.T. Ro, Int. J. Heat Mass Transfer 40 (1997) 2105.

[16] R.U. Bloedner, M. Presia, P. Gille, Adv. Mater. Opt. Electron. 3 (1994) 233.

[17] J. Zhou, M. Larrousse, W.R. Wilcox, L.L. Regel, J. Crystal Growth 128 (1993) 173.

[18] L. Juncheng, J. Wanqi, J. Crystal Growth 183 (1998) 140.

[19] J.C. Brice, P. Capper, C.L. Jones, J.J.G. Gosney, Prog. Crystal Growth and Charact. 13 (1986) 197.

[20] C.W. Lan, D.T. Yang, Int. J. Heat Mass Transfer 41 (1998) 4351.

[21] C.W. Lan, M.C. Liang, J. Crystal Growth 186 (1998) 203.

[22] P.N. Brown, A.C. Hindmarsh, L.R. Petzold, SIAM J. Sci. Comput. 15 (1994) 1467.

[23] J.J. Tuma, Engineering Mathematics Handbook, second ed., McGraw-Hill, New York, 1979, p. 200.

[24] NAG Fortran Library, Mark 15, NAG Ltd., UK, 1991.

[25] J.M. Hyun, W.W. Fowlis, A. Warn-Varnas, J. Fluid Mech. 117 (1982) 71.

[26] G. Muller, A. Ostrogorsky, in: D.T.J. Hurle (Ed.), Handbook of Crystal Growth 2b: Growth Mechanisms and Dynamics, North-Holland, Amsterdam, 1994, p. 812.

[27] S. Chandraselhar, Hydrodynamic and Hydromagnetic Stability, Dover, New York, 1961, p. 83. 